\title{
Impact of Random Dispersion Fluctuations on Two-Pump Fiber Optical Parametric Amplifier Performances
}

\author{
N. Othman ${ }^{1, *}$, K. G. Tay ${ }^{1}$, H. Pakarzadeh ${ }^{2}$, N. A. Cholan $^{1}$, N. S. M. Shah ${ }^{3}$, R. Talib ${ }^{1}$ \\ ${ }^{1}$ Faculty of Electrical and Electronic Engineering, Universiti Tun Hussein Onn Malaysia, Malaysia \\ ${ }^{2}$ Department of Physics, Shiraz University of Technology, Iran \\ ${ }^{3}$ Faculty of Engineering Technology, Universiti Tun Hussein Onn Malaysia, Malaysia
}

Received August 4, 2019; Revised October 6, 2019; Accepted December 12, 2019

Copyright $\bigcirc 2019$ by authors, all rights reserved. Authors agree that this article remains permanently open access under the terms of the Creative Commons Attribution License 4.0 International License

\begin{abstract}
The impact of random dispersion fluctuations on two-pump (2-P) fiber optical parametric amplifier (FOPA) performance in terms of parametric gain, 3-dB amplification bandwidth and saturation power is numerically investigated. The four-coupled amplitude equations which represent the parametric process in optical fiber are solved using the Runge-Kutta method. Based on the results, it was observed that the random dispersion fluctuations reduced the parametric gain, increased the saturation power and had no significant effect on 3-dB amplification bandwidth. The resulted behaviors are mainly due to the changes of phase-matching condition in optical fiber. All in all, the random dispersion fluctuations are potentially limited the 2-P FOPA performances.
\end{abstract}

Keywords Nonlinear Optics, Four-Wave Mixing, Dispersion

\section{Introduction}

Fiber optical parametric amplifier (FOPA) is an optical amplifier that utilizes optical fiber as its amplification or gain medium. The nonlinear effect in optical fiber that makes FOPA possible is the four-wave mixing (FWM). The exploitation of FWM effect has made FOPA useful in various applications, especially in saturation regimes (i.e. large input signal power). The applications in saturation regimes such as all-optical limiters [1], [2], noise suppression [3], signal regeneration [4], [5], pulse regeneration [6], [7] and high-power wavelength conversion [8] have surpassed the limits of conventional optical amplifiers e.g. Raman amplifier and erbium-doped fiber amplifier.
A FOPA is required to show optimum performance in terms of parametric gain and amplification bandwidth. Most applications desire FOPA to achieve high parametric gain and wide amplification bandwidth. However, it is believed that the random dispersion fluctuations can somehow limit the performance of FOPA [9], [10]. In practice, it is impossible to avoid the random dispersion fluctuations in optical fiber since it arises due to the impairment during fabricating process. Therefore, it is vital to consider this event when numerically analyzing the FOPA performance.

Research in [9] and [10] investigated the performance of FOPA in presence of the random dispersion fluctuations. The results exhibited limitation of the FOPA performance when the random dispersion fluctuations were manipulated. Their analyses however were restricted to one-pump (1-P) FOPA. Thus, considering the fact that two-pump (2-P) FOPA also experiences the random dispersion fluctuations in optical fiber, this paper is then focusing on analyzing the impact of dispersion fluctuations on its performance. The impact of dispersion fluctuations was analyzed on the performance of parametric gain, 3-dB amplification bandwidth and saturation power of 2-P FOPA. In this numerical simulation work, both pumps depletion and fiber losses were accounted.

\section{Mathematical Model and Simulation Method}

Parametric process along fiber-length $z$ in 2-P FOPA is modeled by four-coupled amplitude equations of pump $1\left(A_{P 1}\right)$, pump $2\left(A_{P 2}\right)$, signal $\left(A_{s}\right)$ and idler $\left(A_{i}\right)$, such as [11] 


$$
\begin{gathered}
\frac{d A_{P 1}}{d z}=i \gamma\left(\left|A_{P 1}\right|^{2}+2\left|A_{P 2}\right|^{2}+2\left|A_{s}\right|^{2}+2\left|A_{i}\right|^{2}\right) A_{P 1}+ \\
2 i \gamma A_{P 2}^{*} A_{s} A_{i} e^{i \Delta \beta z}-\frac{\alpha}{2} A_{P 1} \\
\frac{d A_{P 2}}{d z}=i \gamma\left(\left|A_{P 2}\right|^{2}+2\left|A_{P 1}\right|^{2}+2\left|A_{s}\right|^{2}+2\left|A_{i}\right|^{2}\right) A_{P 2}+ \\
2 i \gamma A_{P 1}^{*} A_{s} A_{i} e^{i \Delta \beta z}-\frac{\alpha}{2} A_{P 2} \\
\frac{d A_{s}}{d z}=i \gamma\left(\left|A_{s}\right|^{2}+2\left|A_{P 1}\right|^{2}+2\left|A_{P 2}\right|^{2}+2\left|A_{i}\right|^{2}\right) A_{s}+ \\
2 i \gamma A_{i}^{*} A_{P 1} A_{P 2} e^{-i \Delta \beta z}-\frac{\alpha}{2} A_{s} \\
\frac{d A_{i}}{d z}=i \gamma\left(\left|A_{i}\right|^{2}+2\left|A_{P 1}\right|^{2}+2\left|A_{P 2}\right|^{2}+2\left|A_{s}\right|^{2}\right) A_{i}+ \\
2 i \gamma A_{s}^{*} A_{P 1} A_{P 2} e^{-i \Delta \beta z}-\frac{\alpha}{2} A_{i}
\end{gathered}
$$

with $\gamma$ and $\alpha$ denoting optical fiber nonlinearity and fiber losses, correspondingly, while $A_{j}^{*}$ for $j \in\{P 1, P 2, s, i\}$ denotes the complex conjugate of $A_{j}$. The linear phase-mismatch, $\Delta \beta$, is formulated as [11]

$$
\Delta \beta=\beta_{2}\left[\left(\Delta \omega_{s}\right)^{2}-\left(\Delta \omega_{P}\right)^{2}\right]+\frac{\beta_{4}}{12}\left[\left(\Delta \omega_{s}\right)^{4}-\left(\Delta \omega_{P}\right)^{4}\right]+
$$

where $\beta_{2}$ and $\beta_{4}$ are the second-order and fourth-order dispersion coefficients at the central frequency $\omega_{c}=$ $\left(\omega_{P 1}+\omega_{P 2}\right) / 2=\left(\omega_{s}+\omega_{i}\right) / 2$, respectively. Meanwhile, $\Delta \omega_{s}$ and $\Delta \omega_{P}$ can be represented as $\Delta \omega_{s}=\omega_{s}-\omega_{c}$ and $\Delta \omega_{P}=\omega_{P 1}-\omega_{c}=\left(\omega_{P 1}-\omega_{P 2}\right) / 2$.

The $\delta \beta$ term in Eq. (5) represents the random dispersion fluctuations in optical fiber and it can be further resolved as $\delta \beta=\sigma \times n$ [9], [10], [12], with $n$ denoting the normal distribution in the range of $[-1,1]$ and $\sigma$ is the standard deviation. The standard deviation $\sigma$ which is also known as the fluctuations amplitude is described as $\sigma=f \gamma\left[\left(P_{P 1,0}+P_{P 2,0}\right) / 2\right]$, where $f$ is a dimensionless constant, while $P_{P 1,0}$ and $P_{P 2,0}$ are the input powers of pump 1 and pump 2 , respectively.

In this paper, the random dispersion fluctuation along the fiber length is assumed to be a piecewise constant with a correlation length $L_{c}$. In simulation work, a highly nonlinear dispersion-shifted fiber (HNL-DSF) is divided into segments $N$, such as $N=L / L_{c}$, where $L$ is the fiber length. The length of each segment is given as $z=-L_{c} \times$ $\ln (n)$ and over the segment length, the fluctuation is considered fixed.

Now, in order to obtain parametric gain with the influence of random dispersion fluctuations, firstly, Eqs. (1) - (4) with the initial values of $A_{j, 0}=\sqrt{P_{j, 0}}$ for $j \in\{P 1, P 2, s, i\}$ were solved using the Runge-Kutta method for the first segment. Next, by treating the outputs of the first segment as the inputs for the second segment, again Eqs. (1) - (4) were solved. The same process was repeated for the subsequent segments and terminated once the outputs of the last segment were obtained. Upon obtaining the output signal power, $P_{S}$, and with the input signal power, $P_{s 0}$, the parametric gain $G$ then can be calculated as $G=10 \log \left(P_{s} / P_{s 0}\right) \mathrm{dB}$ [13]. Due to the stochastic nature of the dispersion fluctuations, a similar process is simulated for a variation of $\delta \beta$ pattern with the same value of $\sigma$ and $L_{c}$. At the end of the process, the average parametric gain $\bar{G}$ for all those $\delta \beta$ patterns at the respective signal wavelength, $\lambda_{s}=2 \pi c / \omega_{s}$, was calculated. All calculations and simulations in this work were conducted in the programming language of MATLAB.

\section{Results and Discussions}

This paper mainly focuses on 2-P FOPA parametric gain, 3-dB amplification bandwidth and saturation power behaviors in the influence of dispersion fluctuations. Evaluations of the aforementioned parameters' behavior are based on two types of data, i.e. the parametric gain with variation of $\lambda_{s}$ and $P_{s 0}$. The configuration of parameters involved in computing the required data are fixed as follows; $L=500 \mathrm{~m}, L_{c}=100 \mathrm{~m}, f=0.5, \gamma=11.5$ $\mathrm{W}^{-1} \mathrm{~km}^{-1}, \alpha=0.82 \mathrm{~dB} / \mathrm{km}, \beta_{2}=-1.97 \times 10^{-2} \mathrm{ps}^{2} / \mathrm{km}$ and $\beta_{4}=6.23 \times 10^{-5} \mathrm{ps}^{4} / \mathrm{km}$ at central wavelength $\lambda_{c}=1558$ $\mathrm{nm}$. The zero-dispersion wavelength of HNL-DSF is at $\lambda_{0}=1556.5 \mathrm{~nm}$, while pump 1 and 2 are injected at wavelengths of $\lambda_{P 1}=1553 \mathrm{~nm}$ with $P_{P 1}=1 \mathrm{~W}$ and $\lambda_{P 2}=1563 \mathrm{~nm}$ with $P_{P 2}=0.5 \mathrm{~W}$, respectively.

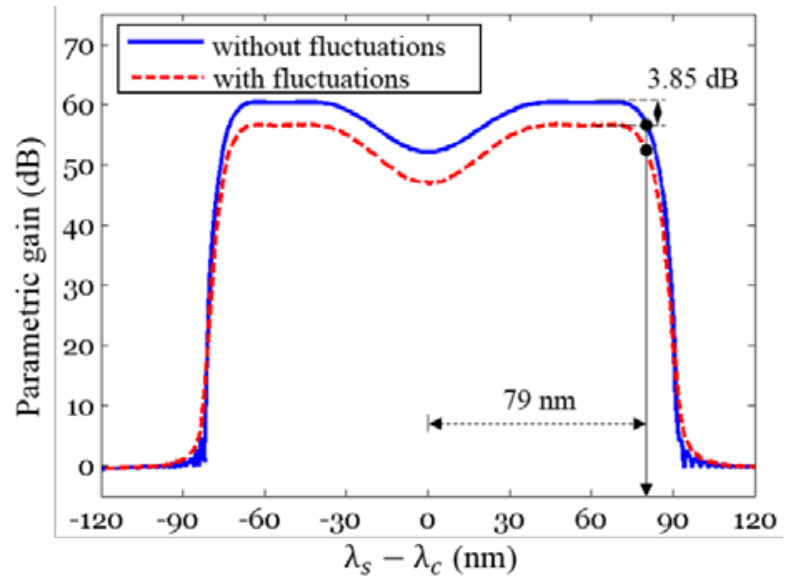

Figure 1. Parametric gain spectra of 2-P FOPA without and with dispersion fluctuations

Firstly, the effect of dispersion fluctuations is observed on 2-P FOPA gain spectrum in terms of parametric gain and 3-dB amplification bandwidth. The 3-dB amplification bandwidth is measured by the wavelength span of which the signal is amplified and the parametric gain is diminished by $3 \mathrm{~dB}$ from the peak value. So now, using the parameters' configuration given before, the parametric gains were computed for a variation of signal wavelength $\lambda_{s}$ and the results are plotted in Fig. 1. Based on the figure, there are two points that are worth to be noted. First, the existence of dispersion fluctuations reduced peak gain of the spectrum. In detail, peak gain of the spectrum without dispersion fluctuations experienced a reduction of about $3.85 \mathrm{~dB}$ once the dispersion fluctuations were taken into account. This is because the existence of dispersion fluctuations in HNL-DSF has affected the phase-matching condition. The phase-matching condition has been 
degraded by the dispersion fluctuations, thus reducing the parametric gain. Second, the dispersion fluctuations exhibit insignificant effect on the 3-dB amplification bandwidth. As seen, the 3-dB amplification bandwidths of both spectra are similar, which is $79 \mathrm{~nm}$. This means that the phase-matching condition over a wide bandwidth is barely affected by the dispersion fluctuations, therefore resulting in almost similar bandwidth.

Now, the impact of dispersion fluctuations on saturation power behavior of 2-P FOPA is investigated. In order to investigate the saturation power behavior, the signal wavelength is fixed at $\lambda_{s}=1615 \mathrm{~nm}$, while the other parameters are configured as before. The results are shown in Fig. 2. Generally, the figure shows that the saturation power (the point at where the parametric gain begins to reduce $3 \mathrm{~dB}$ with respect to the initial value) which are denot--ed by vertical lines, changed over the existence of dispersion fluctuations. Generally, the saturation power is shifted to the larger input signal power once the dispersion fluctuations are considered. Specifically, the saturation power of when the dispersion fluctuations are ignored is $-32.46 \mathrm{dBm}$, and it shifted to the larger input power which is $-30.94 \mathrm{dBm}$ when the dispersion fluctuations are accounted. The saturation behavior in 2-P FOPA can be explained by the power evolution between pump 1, pump 2 , signal and idler; see Fig. 3. The power evolution figures actually explained the power transfer process. Initially, both output pump 1 and pump 2 powers are constant, while the output signal power increases. This behavior is due to the power transfer process from the pumps to the signal. Then, there comes a point where the constant output pumps power begins to decrease, this point indicates that the parametric gain begins to saturate as the input signal power is getting higher. Up to this moment, the powers are still being transferred from the pumps to the signal. However, beyond the point of where the output pumps power is minimum and the output signal power is maximum, the power is now transferred in reversed direction, i.e. from the signal to the pumps. The aforementioned power transfer process was occurred at the higher input power level when the dispersion fluctuations are considered, thus shifting the saturation power to the higher input signal power.

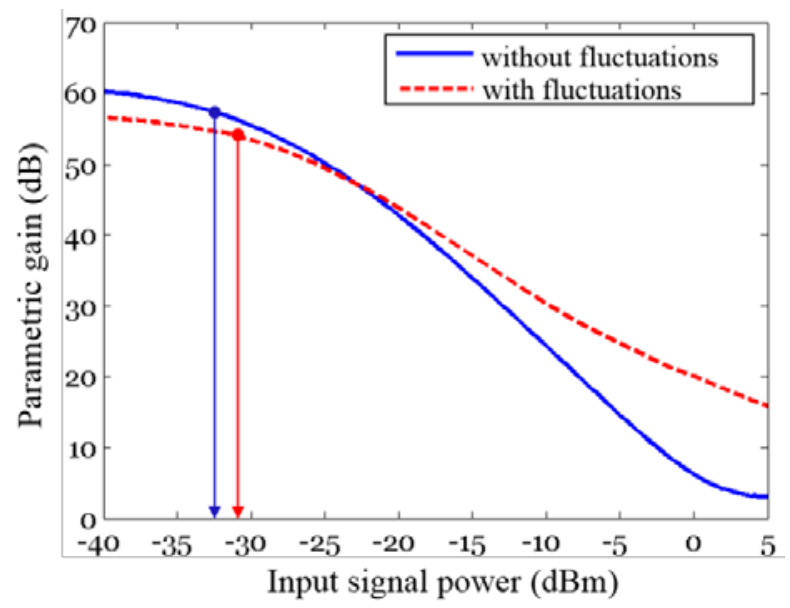

Figure 2. Parametric gain saturation curves of 2-P FOPA without and with dispersion fluctuations at $\lambda_{s}=1615 \mathrm{~nm}$
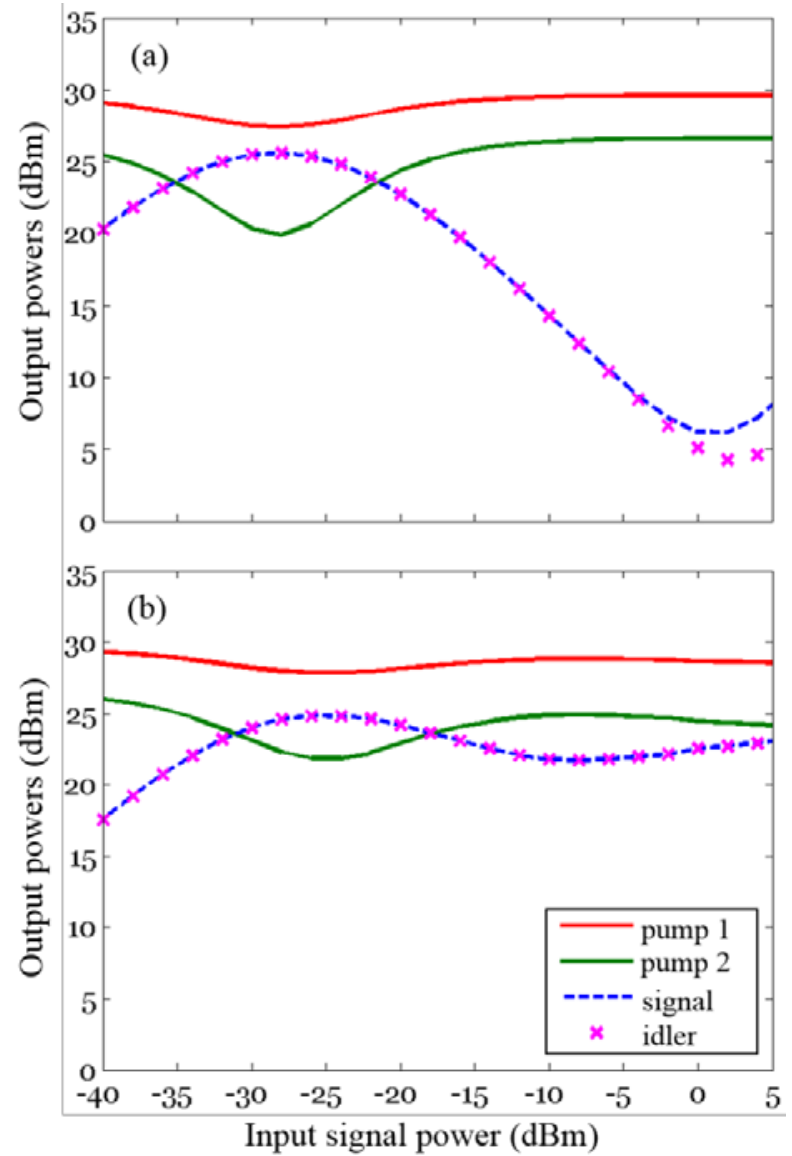

Figure 3. Power evolutions in 2-P FOPA (a) without and (b) with dispersion fluctuations at $\lambda_{s}=1615 \mathrm{~nm}$ 


\section{Summary}

This paper investigated the impact of dispersion fluctuations on 2-P FOPA performance, in terms of parametric gain, 3-dB amplification bandwidth and saturation power. It was observed that the dispersion fluctuations significantly affect the parametric gain and saturation power but not 3-dB amplification bandwidth. Mainly, with the existence of the dispersion fluctuations, the parametric gain was reduced and saturation power at a particular signal wavelength was shifted to the higher input power level. Meanwhile, the performance of 3-dB amplification bandwidth is similar irrespective the dispersion fluctuations. The changes in 2-P FOPA performances when the dispersion fluctuations were taken into account were principally due to the changes in phase-matching condition between the interactive waves along the optical fiber.

\section{Acknowledgements}

This research was supported in part by Fundamental Research Grant Scheme (FRGS) vot K095 granted by Ministry of Education (MOE) Malaysia.

\section{REFERENCES}

[1] K. Inoue. Optical level equalisation based on gain saturation in fibre optical parametric amplifier, Electron. Lett., Vol. 36, No. 12, 1016-1017, 2000.

[2] K. R. H. Bottrill, F. Parmigiani, D. J. Richardson, P. Petropoulos. Optical predistortion enabling phase preservation in optical signal processing demonstrated in FWM-based amplitude limiter, J. Light. Technol., Vol. 35, No. 4, 963-970, 2017.

[3] H. Pakarzadeh, A. Zakery. Modelling of noise suppression in gain-saturated fiber optical parametric amplifiers, Opt. Commun., Vol. 309, 30-36, 2013.

[4] C. Peucheret, M. Lorenzen, J. Seoane, D. Noordegraaf, C.V. Nielsen, L. Grüner-Nielsen, K. Rottwitt. Amplitude regeneration of RZ-DPSK signals in single-pump fiber-optic parametric amplifiers, IEEE Photonics Technol. Lett., Vol. 21, No. 13, 872-874, 2009.

[5] H. Wang, Q. Wang, L. Bai, Y. Ji. All-optical simultaneous multichannel quadrature phase shift keying signal regeneration based on phase-sensitive amplification, Opt. Eng., Vol. 57, 016111, 2018.

[6] D. J. Wilson, X. Ren, S. Zigo, F. Légaré, C. Trallero-Herrero. Simultaneous few-cycle pulse generation of the depleted pump and signal from an optical parametric amplifier, JOSA B, Vol. 35, A45-A48, 2018.

[7] A. Vedadi, A. M. Ariaei, M. M. Jadidi, J. A. Salehi. Theoretical study of high repetition rate short pulse generation with fiber optical parametric amplification, J.
Light. Technol., Vol. 30, 1263-1268, 2012.

[8] Y. Wang, C. Yu, T. Luo, L. Yan, Z. Pan, A. E. Willner. Tunable all-optical wavelength conversion and wavelength multicasting using orthogonally polarized fiber FWM, J. Light. Technol., Vol. 23, No. 10, 3331-3338, 2005.

[9] N. Othman, N. S. M. Shah, K. G. Tay, H. Pakarzadeh, N. A. Cholan. One-pump fiber optical parametric amplifier in presence of dispersion fluctuations: impact of fourth-order dispersion coefficient, Appl. Opt., Vol. 56, No. 29, 8303, 2017.

[10] M. Bagheri, H. Pakarzadeh, A. Keshavarz. Gain-saturated one-pump fiber optical parametric amplifiers in presence of longitudinal dispersion fluctuations, Appl. Opt., Vol. 55, No. 13, 3368-3372, 2016.

[11] M. E. Marhic. Fiber Optical Parametric Amplifiers, Oscillators And Related Devices, Cambridge University Press, UK, 2007.

[12] M. Farahmand, M. de Sterke. Parametric amplification in presence of dispersion fluctuations, Opt. Express, Vol. 12, No. 1, 136-142, 2004.

[13] G. Keiser. Optical Fiber Communication, McGraw Hill, Singapore, 2015. 